\title{
EL LIBRO ELECTRÓNICO: INVARIANZAS Y TRANSFORMACIONES
}

José-Antonio Cordón-García y Carlos A. Lopes

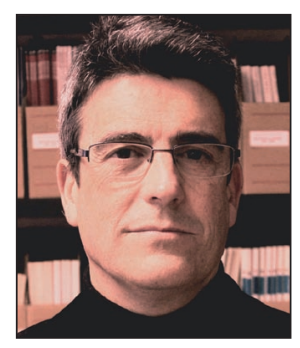

José-Antonio Cordón-García es profesor titular de la Universidad de Salamanca. Miembro del Consejo Ejecutivo del Instituto de Historia del libro y de la Lectura, ha publicado numerosos artículos y monografías sobre la industria editorial en general y el libro electrónico en particular. Dirige el grupo de investigación E-lectra centrado en el análisis de la edición electrónica y las nuevas formas de creación y comunicación escrita. Es director de la revista Pliegos de Yuste: revista de ciencia, cultura y pensamiento europeos, y presidente de la Asociación Española de Bibliología. Forma parte del proyecto Territorio Ebook, de la Fundación Germán Sánchez Ruipérez.

Universidad de Salamanca Facultad de Traducción y Documentación Francisco Vitoria, 6-16. 37008 Salamanca, España http://www.pliegosdeyuste.eu jcordon@usal.es

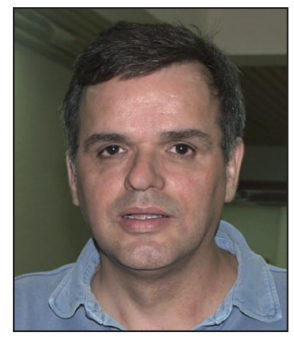

Carlos A. Lopes es director del Centro de Documentación del Instituto Universitário de Ciências Psicológicas, Sociais e da Vida (ISPA). Es profesor del ISPA-IU donde imparte asignaturas relacionadas con la alfabetización informacional (alfin), recursos y comunicación en psicología. Es doctor en biblioteconomía y documentación por la Universidad de Salamanca y por la Universidade de Coimbra. Su trayectoria investigadora se centra en el estudio de la alfin y la calidad en bibliotecas universitarias. Es autor de diferentes artículos en revistas especializadas. Ha formado parte del comité SciELO Portugal y de la Biblioteca do Conhecimento Online (B-on).

Instituto Univ. de Ciências Psicológicas, Sociais e da Vida (ISPA), Centro de Documentação Jardim do Tabaco, 34. 1149041 Lisboa, Portugal http://www.ispa.pt clopes@ispa.pt

\section{Resumen}

Los libros electrónicos han renovado el concepto tradicional de libro, favoreciendo la socialización de la lectura, la participación del usuario y la ruptura de la cadena tradicional de la edición. Las empresas editoriales se han ido adaptando al nuevo contexto con propuestas novedosas, en las que el contenido reviste una importancia cada vez mayor. Se está produciendo una lucha entre los grandes grupos como Amazon, Apple y Google por el control del mercado a medida que éste se consolida. Esas marcas se van internacionalizando y durante 2011 han desembarcado en varios países europeos y latinoamericanos, manteniendo posiciones encontradas.

\section{Palabras clave}

Libros electrónicos, Lectura social, Amazon, Apple, Google.

\section{Title: The e-book: invariances and transformations}

\section{Abstract}

Electronic books have renewed the traditional concept of the book, encouraging the socialization of reading and user participation while breaking the traditional pattern of publishing. The publishing companies have been adapting to the new context with new proposals, in which the content is of increasing importance. There is a struggle between large groups such as Amazon, Apple and Google for the control of the market as it matures. These brands are internationalizing and in 2011 landed in several European and Latin American countries, maintaining their positions as direct competitors.

\section{Keywords}

Ebooks, Social reading, Amazon, Apple, Google.

Cordón-García, José-Antonio; Lopes, Carlos A. "El libro electrónico: invarianzas y transformaciones". El profesional de la información, 2012, enero-febrero, v. 21, n. 1, pp. 83-90. 


\section{El futuro del libro}

Desde que apareció la informática doméstica y se vislumbró la posibilidad de que los textos escritos se reprodujeran en cualquier ordenador, el futuro del libro se convirtió en objeto de debate por parte de autores, editores, comunicólogos, semiólogos, filólogos y todos aquellos que, de manera directa o indirecta, tenían algo que ver con la cultura escrita. Desde el principio la cuestión se planteó como una disyuntiva en la que la exclusividad, oposición o prevalencia cifraba el núcleo polémico: impreso vs digital. La discusión ha llegado hasta nuestros días, en una formulación reductiva que ignora que estamos no frente a un escenario de desaparición sino de cambio de naturaleza. No se trata de un mero traspaso de un formato a otro, en el que el papel permanece de alguna manera como indicio o referente en lo digital. El fundamento de la digitalización no radica en la conversión de obras impresas ya editadas a formato electrónico, sino en la creación de nuevas formas digitales, en muchos casos provenientes de actores diferentes de los implicados en la industria tradicional del libro impreso.

Existen ya numerosas editoriales que trabajan sólo con el formato digital y que marcan las nuevas tendencias de lo que se aventura como el libro del futuro. Entre ellas: Atavist y Byliner en Estados Unidos; Publie.net, Walrus Studio y NumerikLivre en Francia; 24 Symbols, Amphibia y Musa a las 9 en España. Por no hablar de proyectos de integración de soportes y medios como Pottermore, modelo de lo que se ha dado en llamar lectura inmersiva, en la que la confluencia de medios conduce a experiencias de lectura enriquecidas y personalizables. Algunos libros infantiles integran textos con la imagen en movimiento, la interactividad y el sonido, transformándose en experiencias multimedia.

En el universo digital surgen nuevos géneros, desde los más frívolos fan fictions -grupos de entusiastas de una obra o un personaje que continúan una saga o un título en particular para compartirlo entre ellos-, a las wikinovelas, diginovelas, obras colaborativas, obras transmedia..., toda una nueva narrativa basada en las nuevas tecnologías.

Lo que es fundamental es saber distinguir las tendencias de fondo de lo que son meros epifenómenos. Decía HenriJean Martin, en el epílogo de su obra Historia y poderes de lo escrito (Trea, 1999), que la misión de nuestra generación es comunicar a nuestros descendientes que el progreso técnico no implica obligatoriamente el rechazo irreflexivo hacia los soportes del pasado. http://atavist.net http://byliner.com http://www.publie.net http://www.walrus-books.com http://www.numeriklivres.com http://www.24symbols.com http://www.amphibiaeditorial.com http://www.musaalas9.com

El libro es un documento imperecedero, sometido a cambios y transforma-

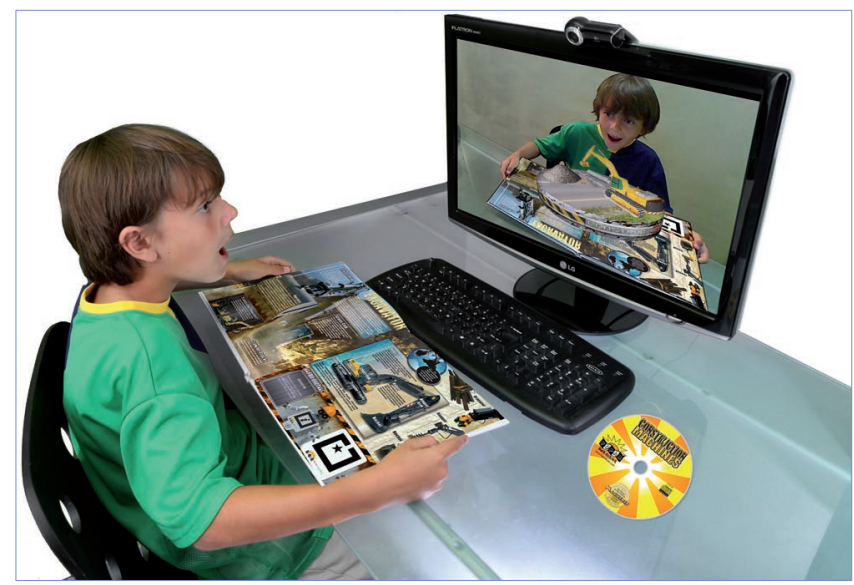

Ejemplo de lectura inmersiva. Fuente: $h t t p: / / w w w . p r w e b . c o m$

ciones permanentes, como han puesto de manifiesto Martin (Trea, 1999), Chartier y Cavallo (Taurus, 2011), Manguel (Lumen, 2005) o Furtado (Trea, 2007, Booktailors, 2011). No hay que olvidar que la forma códice con que ahora lo identificamos tiene sólo 500 años de antigüedad, nada comparado con la era del manuscrito, por ejemplo. Por lo tanto el libro como tal seguirá existiendo como principal vía de comunicación social para pensamientos, reflexiones y discursos que exijan mayor profundidad y extensión.

\section{El fundamento de la digitalización no ra- dica en la conversión de obras impresas a formato digital, sino en la creación de nuevas formas digitales}

La unidad de pensamiento puede ser la frase, el párrafo, la sección o el capítulo de un libro. Pero la forma extensa, una historia, un relato unificado, una discusión completa..., ejerce una fascinación, una extraña atracción sobre nosotros. En la Red es lo mismo: podemos cortar el libro en bits y enlazarlos o tejerlos con otros, pero la organización de primer nivel del libro seguirá centrando la atención.

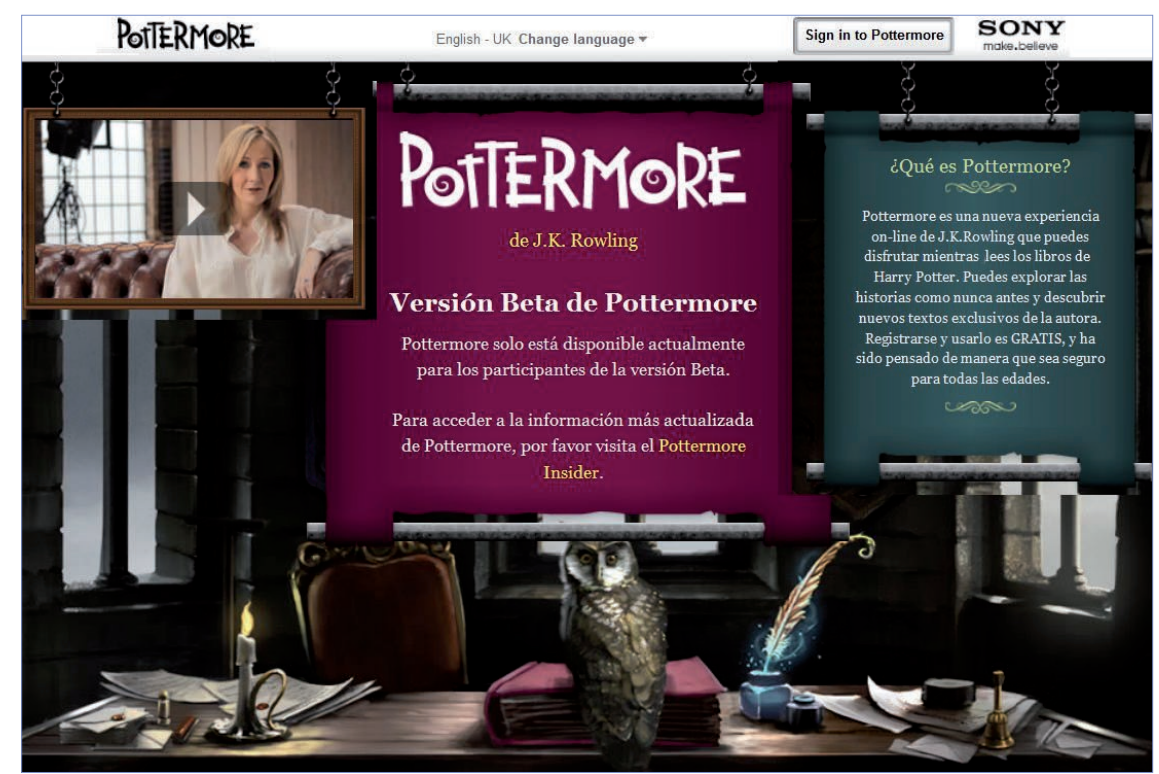

Pottermore es una web para usar mientras se leen libros de Harry Potter, http://www.pottermore.com/es 
Es cierto que su forma cambiará. Las nuevas tecnologías han abierto su condición hermética convirtiéndolo en un medio en el que la integración multimedia, la participación social y la colaboración son cada vez más importantes. Programas de autopublicación como los de Amazon, Barnes \& Noble, Apple, Smashwords, etc.; sistemas de lectura social como aNobii, Rethink Books, Copia, Library Thing, etc., así lo atestiguan.

También algunas obras tradicionales se van transformando: los diccionarios, por ejemplo, se convierten en servicios de tratamiento lingüístico y terminológico.

El papel no es más que una de las posibles versiones de libro que se pueden desplegar y concurrir simultáneamente en el mercado.

El libro tenderá a convertirse en servicio. De objeto se transformará en flujo, escapando al control de sus productores en beneficio de los que los comunican y favorecen su circulación. Un ejemplo de ello son las obras de la editorial Publie.net. Uno de sus títulos, por ejemplo, Après le livre, de François Bon, se puede leer en pdf, en epub, en mobipocket, en streaming, etc., pero la obra a su vez se va actualizando, de tal manera que, publicada en enero de 2011 a día de hoy (octubre de 2011) ha experimentado ya 9 actualizaciones.

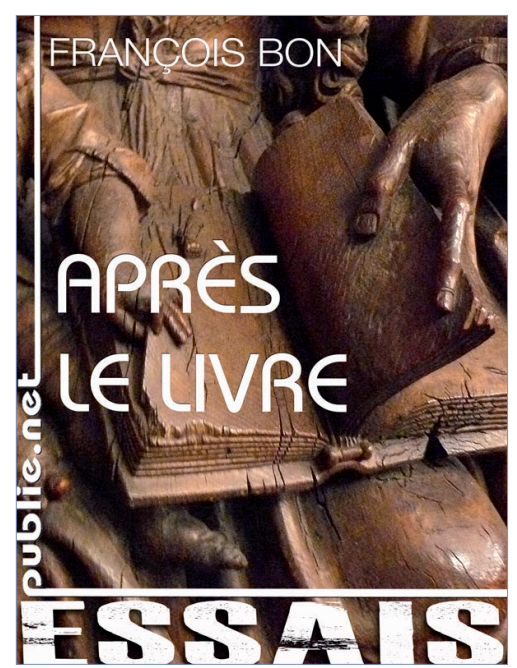

http://www.publie.net/fr/ebook/97828145 04103
Por otra parte los lectores están cada vez más involucrados en el proceso de las obras mediante sistemas que interactúan con los autores, como@author de Amazon que permite comentar pasajes del libro con sus creadores, lo mismo que ocurre con el programa Book country de Penguin, produciéndose una interactividad antes imposible. También se dan versiones personalizadas de las obras, anotaciones y una serie de actividades que antes formaban parte de las prerrogativas exclusivas del autor y el editor. Además, las propias aplicaciones de lectura incluyen ya la posibilidad de socialización de la lectura y personalización de la misma de una manera intuitiva para el usuario. Tal es el caso de iBooks, la aplicación de lectura del iPad, o de Stanza o de Kobo. La experiencia de lectura se hace cada vez más colaborativa y abre unos horizontes a la misma, vinculando la experiencia personal a servicios en red como los de ReadMill o Amazon.

Por lo tanto apreciamos que no sólo cambia la estructura del mercado, sino el mismo concepto de libro, que ha evolucionado en los últimos 40 años de la mano de dos personas reciente y prematuramente fallecidas ${ }^{1}$, Michael Hart, creador del Proyecto Gutenberg en 1971, el primer portal dedicado a la digitalización de obras cuando en Occidente comenzaba a desarrollarse la informática, y Steve Jobs, fundador de Apple y creador de ingenios como el iPod o, más recientemente el iPad.

\section{Impacto de los libros electrónicos en España: tendencias actuales y futuras}

En España la producción de libros electrónicos ha experimentado un crecimiento progresivo, siendo del $155 \%$ en el año 2010 (Panorámica, 2011). Frente a los 12.514 ISBNs concedidos en 2009, en 2010 han sido 18.500, producidos por pequeñas y medianas editoriales que aglutinan el $60 \%$ del mercado, y con un significativo $8,2 \%$ relativo a la autoedición (casi un 30\% con respecto al año 2009).

Las cifras de lectura no van a la zaga. Según Hábitos de Lectura y Compra de Libros (2011) en 2010 cerca de la mitad de la población de 14 o más años $(47,8 \%)$ es lectora de formato digital. De ésta, un porcentaje todavía elevado (48\%) lee en el ordenador, un 6,6 en el móvil, y un $0,8 \%$ en el ereader, porcentaje que se eleva al $1,1 \%$ en la encuesta del 2o cuatrimestre de 2010. El perfil de los lectores digitales es interesante porque ilustra acerca de las tendencias y los desarrollos futuros.

Una evidencia de esta progresión es que en los datos aportados para el primer semestre de 2011, la lectura digital ha pasado de $47,8 \%$ al $52,5 \%$. Así pues los lectores en soporte digital han crecido en los primeros 6 meses del año 4,7 puntos respecto al año 2010. En libros electrónicos, 2,1 puntos respecto al $1^{r}$ cuatrimestre de 2010 y 1,5 puntos respecto a la media del pasado año.

La industria editorial también está cada vez más involucrada en este proceso. Según el último informe El libro digital en España (Federación de Gremios de Editores de España, 2011) el $75 \%$ de los editores ha elaborado planes de digitalización de su catálogo. Sin embargo en España se podría hablar de una cierta dicotomía entre el funcionamiento del mercado y el de la sociedad, en el sentido de que mientras que la recepción por parte de ésta es cada vez más importante, como demuestran las cifras de venta de dispositivos de lectura electrónica, la actitud de los editores evidencia una excesiva prudencia, constatable en el hecho de que la colección de obras electrónicas disponibles es todavía escasa en relación con el nivel del sector, cuarto en importancia en el mundo.

\section{En España los usuarios son cada vez más receptivos, pero los editores son excesi- vamente prudentes}

Los editores temen la posibilidad de canibalización del libro impreso por el digital, sin entender que lo que se está produciendo es una oportunidad de vender que, de cualquier modo, reviste la contundencia de los movimientos de carácter inexorable. No se trata ya de una opción que se pueda elegir o no, sino de una exigencia del mercado. Y así se ha puesto de manifiesto en los foros profesionales como Liber, en el que la edición digital ha sido la protagonista en los 
últimos años. O en el último Ficod (Foro internacional de contenidos digitales) celebrado en Madrid en noviembre de 2011, en el que se presentó el informe del Observatorio Nacional de las Telecomunicaciones y la Sociedad de la Información (Ontsi, 2011), según el cual en 2010 por primera vez se habían consumido en España más contenidos digitales que impresos. Los informes de The Cocktail Analysis (2011) y de The New Media Consortium (The horizon report, Johnson et al., 2011), entre otros, confirman esta tendencia hacia el consumo de contenidos digitales, en la que las pantallas alcanzan un protagonismo singular, con tablets $y$ smartphones como punta de lanza, y en la que la lectura de libros reviste cada vez más importancia (ComScore, 2011). http://en.www.mcu.es/libro/MC/ObservatorioLect/ Estudios/estudiosOtros/NuevasTec.html http://www.tcanalysis.com

La progresión es permanente y será cada vez más importante en la medida en que empresas como 24 Symbols -que propone la distribución de libros electrónicos en la nube con sistemas freemiun (gratuitos pero con publicidad no invasiva) o premium (sin anuncios)- se vayan consolidando. Cada vez hay más propuestas editoriales, y únicamente falta que el mercado madure con unos precios más aquilatados, y que vayan siendo mayoría las jóvenes generaciones de nativos digitales, que constituyen un motor de desarrollo.

Los movimientos de los editores evidencian una implicación cada vez más importante en lo digital que no sólo afecta a las obras de ficción sino que tiene ramificaciones en el libro científico, y en el libro infantil y juvenil. Precisamente en este último sector se han generado importantes desarrollos para tablet principalmente por parte de Oceanhouse Media, Ruckus Media, Smashing Ideas, y Trilogy Studios. Valorando la importancia de este emergente sector digital, Random House compró recientemente al programador de Smashing Ideas. Una muestra de la importancia que va cobrando la constituye el hecho de que en la última feria del libro de Francfort se dedicara un panel específico a la edición infantil con el significativo título de Children's publishing goes digital. Pero en el resto de los sectores se multiplican igualmente propuestas novedosas provenientes de actores muy diversos, como puede ser un medio de comunicación como La vanguardia, que en noviembre de 2011 lanzó su propia editorial de libros electrónicos, Ebooks de vanguardia, con varios titulos ya en cartera y colecciones como Periodismo de vanguardia: más de un centenar de reportajes y series de artículos de $L a$ vanguardia publicados en colaboración con Amazon con motivo del lanzamiento de la tienda Kindle en España el 1 de diciembre de 2011. La mayor parte de títulos cuestan 0,94 euros.

Ediciones $B$ ha lanzado también su propia propuesta editorial, una colección de libros sin DRM, contraviniendo la tendencia mayoritaria seguida por el sector editorial de protección de obras mediante este sistema. Se trata de la colección $B$ de Books con más de cien títulos ya en el mercado a precios en muchos casos inferiores a los 2 euros.

La editorial Debate recurre a la fórmula de lectura en streaming. En 2011, lanzó Endebate, una colección sólo digital de textos de 10.000 palabras, disponibles tanto para iPad como en formato epub.

\section{El poder del libro de ofrecer una historia autónoma, un relato unificado, una dis- cusión completa..., ejerce una extraña atracción sobre nosotros}

El grupo Planeta, por su parte, lanzó igualmente a finales de 2011 Scyla y Zafiro, dos nuevos sellos editoriales creados para publicar exclusivamente eBooks. Scyla está orientada a la ciencia ficción, la fantasía y el terror en tanto que Zafiro se ocupa del género romántico

http://www.oceanhousemedia.com/products

http://www.ruckusmediagroup.com

http://smashingideas.com

http://www.trilogystudios.com

\section{Internacionalización del sector: edición comercial y edición académica}

Desde hace muchos años la edición se ha caracterizado por su tendencia a la internacionalización y a la concentración de empresas. Los grandes conglomerados editoriales tienen vocación de monopolio, y esto es un axioma para grupos pre-electrónicos como Bertelsmann, Elsevier, Hachette, Planeta, etc.

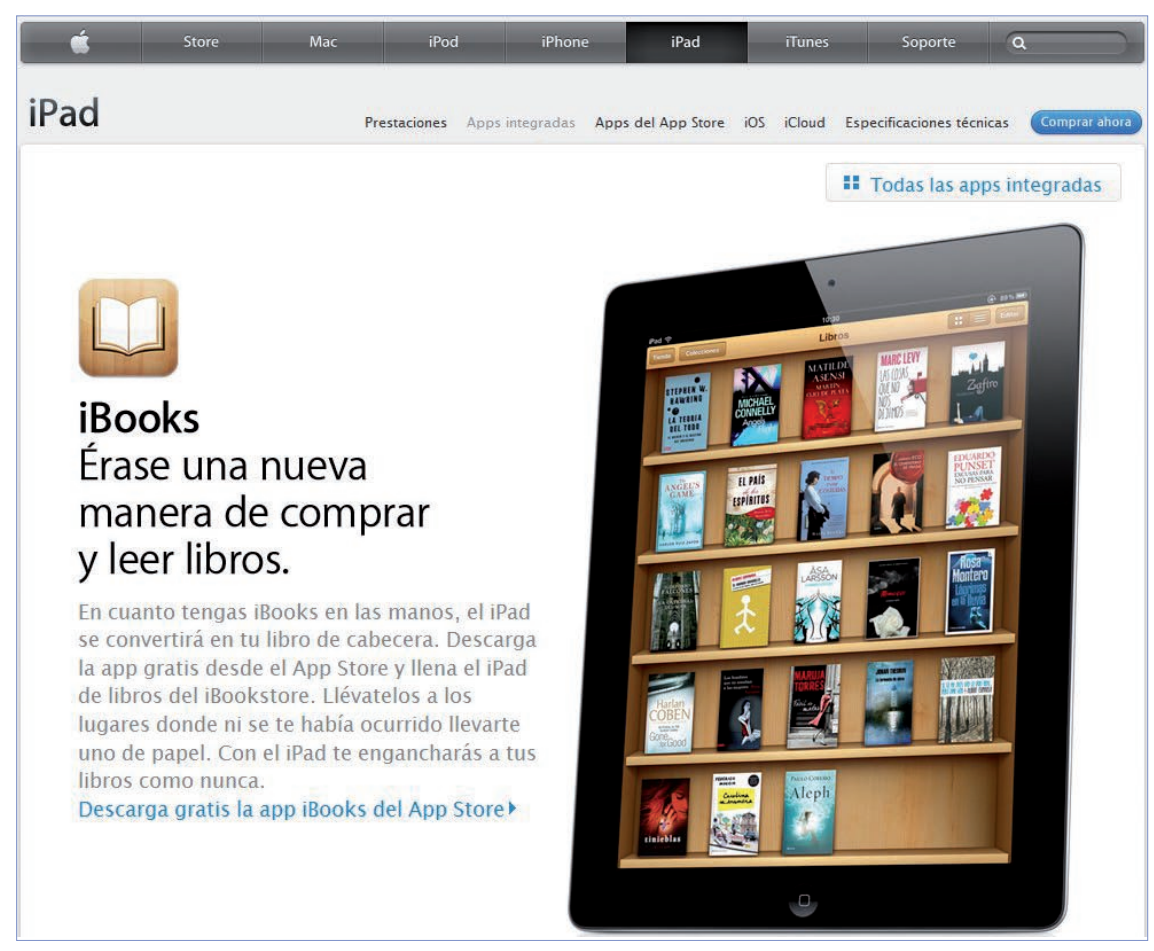

Web iBookStore de Apple, http://www.apple.com/es/ipad/built-in-apps/ibooks.html 
La edición electrónica en general y los libros electrónicos en particular, no son una excepción, y debido a la globalización dicha tendencia se ha acentuado. Los sistemas de integración vertical como los llevados a cabo por Apple o por Amazon tienen la misma orientación de acaparar mercado. Apple lo ha demostrado con su política de admisiones y rechazos de firmas de la competencia en las apps de iBookStore. Google, Amazon y Apple han iniciado una política de expansión territorial que competirá en el ámbito lingüístico, un terreno en el que tienen todas las ventajas de las sinergias generadas desde sus plataformas. Esta expansión se ha materializado en el desembarco de Amazon en España en septiembre de 2011, lo mismo que hizo Apple que abrió su iBookStore en ese mismo mes, con la particularidad de que importantes grupos editoria-

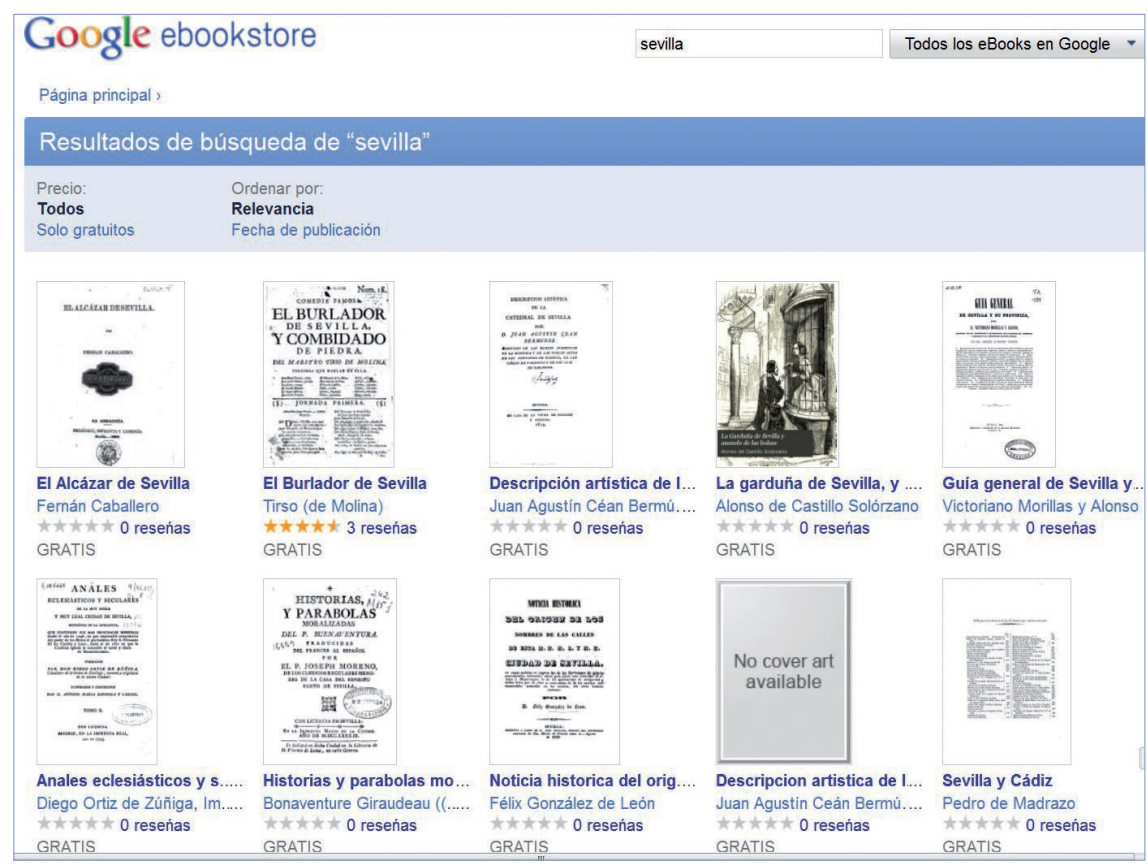

A finales de 2011 en la web eBookStore sólo se ofrecían gratuitamente los libros escaneados por Google cuyos derechos de autor han caducado, más de 2 millones. http://books.google.es/ebooks les como Planeta, la empresa multimedia y de edición más importante de España, está incorporando su catálogo a la tienda de Apple. Además estarán las obras de Seix Barral, Destino, Espasa, Crítica, Paidós y Mondadori. El fondo de 400.000 obras con que cuenta iBookStore se verá incrementado con una mayor disponibilidad de títulos en castellano.

La irrupción más desequilibrante en España puede ser la de Google eBooks, aunque se ha retrasado y se anuncia ahora para principios de 2012

Sin embargo, con ser importantes estos movimientos, la irrupción más desequilibrante puede ser la de Google eBooks. Google cuenta con un catálogo de varios millones de títulos y anuncia unos descuentos en el precio de casi el $80 \%$ sobre los libros en papel. Se dará, por tanto, una auténtica pugna por la conquista del mercado. Google Books ha acostumbrado a sus usuarios a buscar y encontrar millones de títulos difíciles de localizar en formato papel. Los ha acostumbrado a descubrir citas o referencias en un corpus textual ingente, y ha elevado el libro a la misma condición que las noticias, sitios web y otros servicios propios de internet. Con ello ha ayudado a popularizar un tipo de documento propio de la biblioteca y de la librería, y ha instaurado un hábito muy interesante para el paso siguiente: la creación de Google eBooks, transformando su plataforma en vendedora y distribuidora de libros. La facilidad de uso, la variedad de posibilidades y la existencia de un catálogo ingente de títulos aventuran una seria competencia para el resto de empresas del sector. Google tiene además la ventaja añadida de la complementariedad con el resto de sus servicios, que permitirá la integración e implementación de Google eBooks con sistemas de búsqueda y redes sociales.

http://books.google.com/ebooks
No hay que olvidar la derivación latinoamericana del mercado de libros en español, hasta ahora en cierto modo estable, gracias al efecto proteccionista del precio fijo del libro, al que están obligados todos los actores que intervienen en España. Esto dificulta una competencia real, pero previsiblemente la situación irá cambiando en la medida en que las leyes de libre competencia afecten al sector del libro. También afectará la introducción del IVA reducido, pues el actual (del $18 \%$ en todos los países europeos) le resta competitividad en un mercado cada vez más globalizado.

De cualquier modo lo que es una realidad es la presencia de Amazon en España con una oferta de títulos cada vez más abundante y una política de precios agresiva que se ha hecho extensiva a la venta de su dispositivo estrella, el Kindle. A finales de diciembre de 2011, tan sólo tres meses y medio después de su apertura, Amazon tenía ya 2,2 millones de usuarios únicos mensuales, la mitad de los que poseía el líder español El Corte Inglés. La industria editorial española se está posicionando a través de la creación de sellos y plataformas con propuestas que pueden ser competitivas si consiguen consolidar una oferta atractiva en contenidos, precios y experiencia de usuario.

Amazon no es sólo una empresa, sino que constituye un ecosistema en el que la oferta de títulos tiene su correlato en la existencia de unos dispositivos de lectura con una relación calidad/precio considerablemente buena. El lanzamiento en septiembre de 2011 de la primera tablet de la marca, el Kindle Fire, representa otro importante paso en la conquista del entorno global de mercado de los libros electrónicos por parte de esta empresa.

http://www.amazon.es

En este contexto internacional la edición académica es un nicho de mercado en constante progresión, abocada inevitablemente a su transformación digital. Las universidades que no lo entiendan así quedarán rezagadas y perderán el tren de la modernidad. Ya ocurrió con las revistas científicas y ocurrirá de nuevo con los libros. 


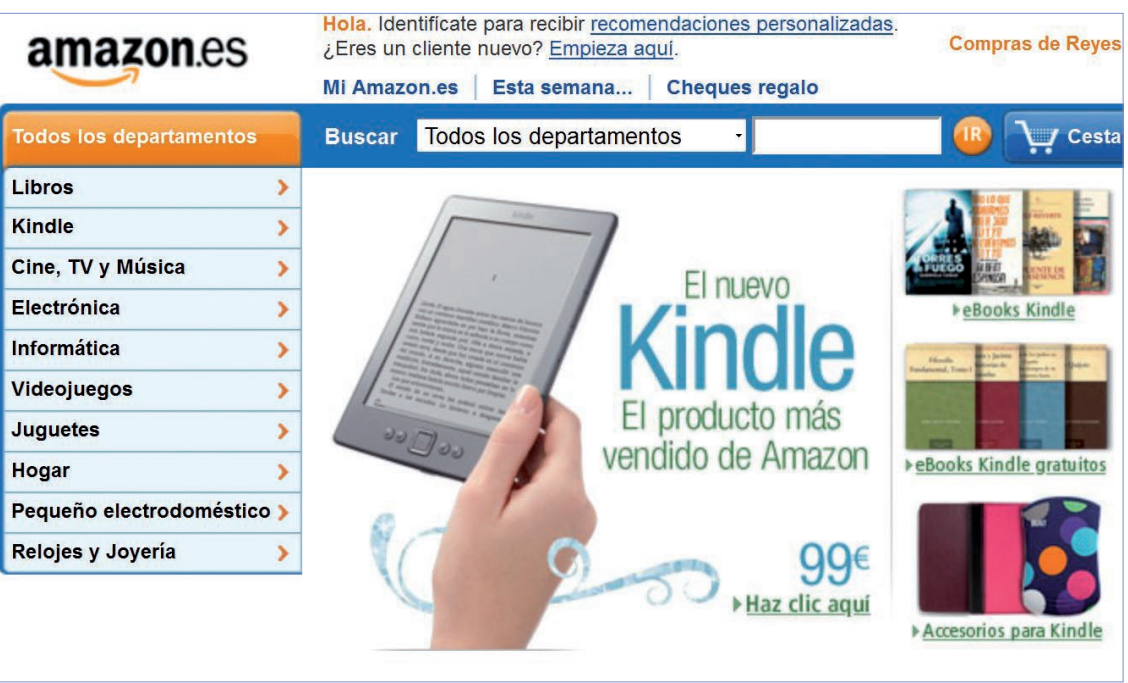

Amazon vende casi de todo en sus webs y los libros han pasado a ser un producto secundario. Su principal fuente de ingresos es la venta de alojamiento (hosting) gracias a su potente y fiable estructura informática. http://amazon.es

Las iniciativas de edición científica no dejan de proliferar. En octubre de 2010 en España se creó el portal Unebook, que agrupa la producción electrónica de todas las editoriales universitarias españolas.

http://www.unebook.es

En los EUA, Jstor, servicio de suscripción de revistas académicas, dio a conocer sus planes para libros electrónicos durante la reunión de enero de 2011 de la American Library Association (ALA). Firmó acuerdos con 5 de las editoriales universitarias más importantes: Chicago, Minnesota, North Carolina, Princeton y Yale para poner sus libros online a lo largo de este año 2011.

http://about.jstor.org/books

Mientras tanto, el proyecto MUSE, que al igual que Jstor suministra acceso a revistas académicas, ha creado una plataforma denominada Project MUSE Editions para distribuir online los libros electrónicos de docenas de editoriales; está previsto que funcione en enero de 2012.

Otro grupo de editores, entre ellos New York University Press, Rutgers University Press, Temple University Press y University of Pennsy/vania Press, ha elaborado un programa colaborativo de plataforma digital para libros denominado University Press e-Book Consortium (UPEC).

En marzo de 2011 UPEC y Project MUSE Editions anunciaron su fusión, creando el University Press Content Consortium (UPCC).

http://muse.jhu.edu/about/new/ebook_collections.html

Open Access Publishing in European Networks (Oapen) es un proyecto lanzado en octubre de 2010 después de 2 años de elaboración, que tiene la particularidad de distribuir los contenidos en acceso abierto. Los derechos varían en función de las distintas políticas editoriales, aunque la mayoría de las obras están bajo licencia Creative Commons. Para la selección de las obras cuenta con un sistema de revisión por pares.

http://www.oapen.org

La Association of American University Presses (AAUP) hizo público en marzo de 2011 un estudio sobre la situación de la digitalización en las diferentes editoriales asociadas. Se trata de una encuesta para conocer qué estrategias se siguen y en qué medida están adoptando el formato digital para las publicaciones académicas. Todas las editoriales universitarias están Ilevando a cabo al menos un par de proyectos con el fin de tener criterios para definir su futura estrategia digital.

Las iniciativas en el área del libro científico no se quedan ahí sino que surgen nuevos modelos de negocio para nuevas formas de lectura. Por ejemplo, CourseSmart, la plataforma de distribución y comercialización de las principales editoriales de libros de texto en Estados Unidos, ha implementado una versión para acceder a sus contenidos a través del iPad. También acaba de actualizar sus versiones para iPhone y iPod touch. Desde el iPad ya es posible el acceso a más de 14.000 libros de texto digitales de cinco grandes editoriales especializadas con descuentos de hasta el $60 \%$ del precio de las ediciones impresas. Hoy ya son 15 las editoriales que colaboran con este proyecto. Su idea es tratar de mejorar la enseñanza y el aprendizaje, proporcionando a los profesores y estudiantes una mejor exposición y acceso a los materiales del curso mediante el formato digital online, y ahora también mediante el soporte móvil. Las tecnologías digitales permiten la creación de comunidades de aprendizaje presencial y virtual. Apple ha creado Inkling para la introducción de libros de texto en el iPad. http://www.inkling.com

El mero acto de entrega de contenido digital no es suficiente. Los editores de hoy necesitan ir más allá con el fin de alcanzar formas innovadoras para mejorar la adquisición de libros electrónicos y para cumplir con las expectativas de los usuarios en cuanto a comodidad, personalización y flexibilidad. Los modelos de alquiler y suscripción de obras académicas caminan en esa línea. Ejemplos son eCampus. com y Cengage que además del alquiler permiten la compra de capítulos y de paquetes de aprendizaje con materiales complementarios.

http://www.ecampus.com

http://www.cengage.com

La integración multimedia, la participación social y la colaboración son cada vez más importantes en el entorno del libro

Proliferan los programas que permiten compartir e intercambiar lecturas científicas y de todo tipo como Copia, la plataforma de lectura digital con mayor enfoque social, que firmó un acuerdo con The Collegiate Retail Alliance (CRA), la mayor coalición de tiendas universitarias independientes de Estados Unidos. Esta coalición ya había llegado anteriormente a otros acuerdos con CaféScribe y CourseSmart para potenciar y satisfacer la demanda de libros electrónicos en las universidades. En marzo de 2011 Copia comenzó un pro- 
grama piloto con 10 librerías de universidades para ofrecer contenidos digitales con varias funciones: compartir notas, grupos de discusión online... Copia tenía previsto implantarse en España en otoño de 2011, incorporando en sus catálogos los libros que comercializan Libranda, Publidisa y Bubok, con una oferta global de 60.000 títulos, que va de los best sellers y las novedades literarias a los libros técnicos.

http://www.thecopia.com/home/index.html

http://www.collegiateretailalliance.com

http://www.libranda.com

http://www.publidisa.com

http://www.bubok.com

\section{Apocalípticos, integrados y asimilados}

En junio de 2011, el periodista y filósofo alemán Florian Rötzer hizo este comentario:

"Para los jóvenes que han crecido en la era de la información, la privación siquiera por un día del acceso a internet se traduce en síntomas de abstinencia física y psíquica similares a los que tienen los fumadores cuando dejan de fumar. Esto se conoce como trastorno de privación de información (information deprivation disorder) y los que lo sufren se sienten excluidos y desconectados del mundo. La función de los espacios públicos y semipúblicos ha comenzado a cambiar. Ya no evolucionamos ni solos ni en grupo, sino en medio de una nube de contactos, amigos e información, a la que a menudo damos más importancia que a nuestro entorno físico inmediato".

La sociedad va creando su ecosistema según va evolucionando. Cuando se recibe un impacto tan grande como el de internet se precisa un tiempo para que los individuos se adapten al mismo. La asimilación de los cambios depende de los niveles de educación, económicos, etc.

Se habla de los posibles efectos perniciosos de la Red, pero, si se compara, la televisión ha sido mucho más devastadora, pues ha impuesto una actitud pasiva y acrítica. Al menos la Red obliga a la actividad y la participación, y que ésta no sea obligatoriamente presencial representa una gran ventaja sobre todo para aquellos que viven en urbes desconectadas. La Red ha abierto canales de comunicación inimaginables hace unos años. Pensemos en las revueltas de los países árabes, o lo que ocurre en dictaduras como China o Cuba donde gracias a la Red la juventud ha adquirido un grado de concienciación y movilización imposibles de otra manera.

La Red no tiene los efectos perniciosos de la TV, que ha sido devastadora al propiciar una actitud pasiva y acrítica

Por lo tanto los vaticinios apocalípticos carecen de sentido, y en la historia se han demostrado siempre exagerados. Los beneficios compensan con creces los inconvenientes que puedan tener. $Y$ el aislamiento y la introversión son síntomas de problemas preexistentes que el uso de la Red pone de manifiesto pero que no han sido generados por ella.

Los libros representan desde siempre el espíritu de la libertad y el progreso, independientemente de los soportes a los que

\section{DER TAGESSPIEGEL Medien}

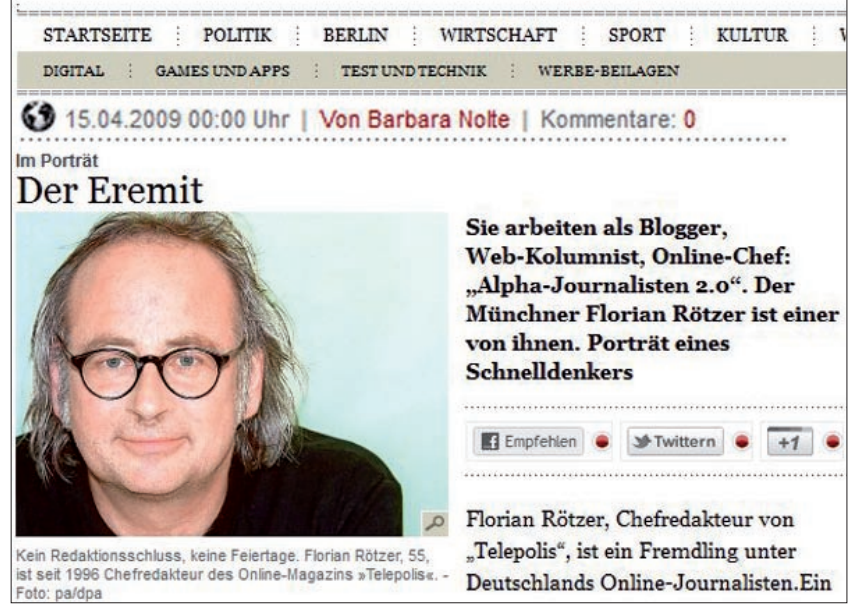

Artículo sobre Florian Rötzer en el periódico Der Tagesspiegel

http://www.tagesspiegel.de/medien/im-portraet-der-eremit/1496438. html

estén asociados. Evidentemente el progreso científico e industrial nos hace cada vez más deudores y dependientes del contexto tecnológico y energético. Pero ¿̇existe una realidad separada del mismo? Hoy en día sería impensable excepto para los integristas de pensamiento radical anclados en propuestas ludistas o misoneístas. Nuestra realidad es tecnológica, imaginativa y en estado de innovación permanente, no sólo en el ámbito del pensamiento o de la cultura en general sino en los aspectos más cotidianos de la vida. La vuelta a la realidad propiciada por una escasez de energía sería una vuelta al paleolítico, no un escenario bucólico o rousseiano de vuelta a la naturaleza, sino apocalíptico como el representado en películas como Mad Max y sus derivados.

La realidad es que la composición de átomos va decayendo en beneficio de los bits, por utilizar la conocida expresión de Negroponte en Being digital.

En septiembre de 2011 se publicó en Idboox.com esta crónica futurista, algo catastrófica:

- 2013: Las ventas de libros electrónicos superan todas las ventas de libros, incluso los libros usados. Las ventas de revistas digitales comienzan a igualarse a las de las revistas impresas.

- 2014: Las editoriales subvencionan aparatos lectores de libros digitales. Los editores de periódicos, revistas y libros tratan de crear lectores bloqueados para vender en ellos sólo sus productos. Fallan.

- 2015: Muerte de las librerías locales. Las más pequeñas se convierten en cibercafés. Sobreviven marginalmente las especializadas en libros raros o de coleccionista.

- 2016: Muchas de las revistas de kiosko se publican sólo en digital.

- 2018: La última tienda de Barnes \& Noble se transforma en cibercafé y en punto de acceso wifi.

- 2019: Hecatombe entre los editores: sólo sobrevive un pequeño número de ellos. Los gigantes como Random House y Penguin transforman sus filiales más pequeñas en editores exclusivamente digitales.

- 2020: Casi todos los estudiantes de colegios y escuelas secundarias poseen un lector de libros electrónicos. Los libros de texto en papel desaparecen gradualmente. 


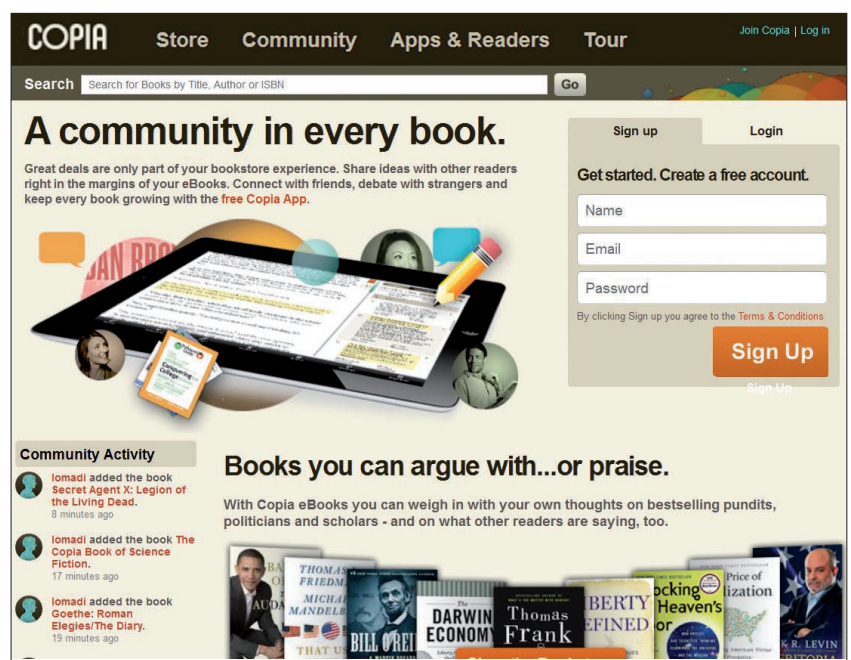

http://www.thecopia.com

- 2023: Los dispositivos de lectura llegan a ser tan delgados como el papel gracias a la evolución del Epaper.

- 2025: Termina la transición de los libros impresos a los electrónicos, incluso en los países en vías de desarrollo. En el mejor de los casos el libro se considera una curiosidad y en el peor una molestia. Los libros de colección aún no han desaparecido. Un pequeño número de lectores sigue leyendo libros impresos, pero en general todos los libros están disponibles en versión digital.

Puede parecer una previsión demasiado apocalíptica pero no está exenta de posibilidades, tal y como marcha el mercado en la actualidad.

Nuestra realidad es tecnológica, imaginativa y en estado de innovación permanente en el ámbito del pensamiento, de la cultura en general y en los aspectos más cotidianos de la vida

\section{Nota}

1. Michael Hart (63) y Steve Jobs (56) fallecieron con un mes de diferencia, 7 de septiembre y 7 de octubre de 2011, respectivamente. Habían alumbrado algunas de las ideas más innovadoras del último siglo.

\section{Bibliografía}

Association of American University Presses (AAUP). Digital book publishing in the AAUP community, Survey report: Spring 2011, 11 pp.

http://www.aaupnet.org/images/stories/data/2011digital surveyreport.pdf

Association of American University Presses (AAUP). Sustaining scholarly publishing: new business models for university presses, March 2011, 37 pp.

http://www.aaupnet.org/images/stories/documents/aaup businessmodels2011.pdf

Chartier, Roger; Cavallo, Guglielmo (eds). Historia de la lectura en el mundo occidental. Madrid: Taurus, 2011, 566 pp. ISBN 9788430608386
The Cocktail Analysis. Televidente 2.0, 2011.

http://www.tcanalysis.com/2011/09/21/televidente20-2011-tablets-television-conectada-y-redes-socialesenriquecen-el-escenario-de-consumo-de-television/

ComScore. Digital omnivores: How tablets, smartphones and connected devices are changing U. S. digital media consumption habits. ComScore, 2011, 33 pp. http://www.ipmark.com/pdf/Omnivoros.pdf

Cordón-García, José-Antonio. La revolución del libro electrónico, Colección El profesional de la información. BarceIona: UOC, 104 pp. ISBN 9788497884853

Federación de Gremios de Editores de España; Fundación Germán Sánchez Ruipérez. $2^{a}$ Encuesta sobre el libro digital en España. Impacto de la digitalización en el catálogo, canales de distribución y de venta y política comercial. Resultados de la encuesta 2010. Informe elaborado por Neturity. Marzo 2011, 45 pp. Disponible en: Distribuidor de Información del Libro Español en Venta (Dilve.es)

http://www.dilve.es/dilve/getArchivoDocumentacion. do?iddocumento $=1541$

Furtado, José-Afonso. El pixel y el papel: de lo impreso a lo digital: continuidades y transformaciones. Gijón: Trea, 2007. 109 pp. ISBN 9788497043007

Furtado, José-Afonso. A edição de livros e a gestão estratégica. Lisboa: Booktailor, 2011. 325 pp. ISBN 978989 9600812

Hábitos de lectura y compra de libros. Madrid: Federación de Gremios de Editores, 2011.

Idboox. Ebook: le futur du livre papier, chronique d'une mort annoncée, 28 septembre 2011.

http://www.idboox.com/ebook/infos-ebooks/ebook-lefutur-du-livre-papier-chronique-d-une-mort-annoncee

Johnson, Larry; Smith, Rachel S.; Willis, Holly; Levine, Alan; Haywood, Keene. The 2011 horizon report. Austin, Texas: The New Media Consortium, 2011. 40 pp. ISBN 978 0982829059

Manguel, Alberto. Una historia de la lectura. Barcelona: Lumen, 2005. 624 pp. ISBN 8426415253

Martin, Henri-Jean. Historia y poderes de lo escrito. Gijón: Trea, 1999. 526 pp. ISBN 8495178400

Negroponte, Nicholas. Being digital, New York: Alfred A. Knopf, 1995, 243 pp ISBN 0679439196

Ontsi (Observatorio Nacional de las Telecomunicaciones y la Sociedad de la Información). La sociedad en red, 2010: informe anual, edición 2011.

http://www.ontsi.red.es/informes-anuales/articles/ id/5421/informe-anual-2010-edicion-2011.html

Panorámica de la edición española de libros 2010. Madrid, Ministerio de Cultura, 2011.

Rötzer, Florian. "La réalité augmentée ou la quête d'un nouveau monde". Courier international, 16-06-2011. http://www.courrierinternational.com/article/2011/06/16/ la-realite-augmentee-ou-la-quete-d-un-nouveau-monde 